\title{
Diabetic Choroidopathy: Choroidal Vascular Density and Volume in Diabetic Retinopathy With Swept-Source Optical Coherence Tomography
}

\author{
JAY C. WANG, INÊS LAÍNS, JOANA PROVIDÊNCIA, GRAYSON W. ARMSTRONG, ANA R. SANTOS, PEDRO GIL, \\ JOÃO GIL, KATHERINE E. TALCOTT, JOÃO H. MARQUES, JOÃO FIGUEIRA, DEMETRIOS G. VAVVAS, \\ IVANA K. KIM, JOAN W. MILLER, DEEBA HUSAIN, RUFINO SILVA, AND JOHN B. MILLER
}

- PURPOSE: To compare choroidal vascular density (CVD) and volume (CVV) in diabetic eyes and controls, using en face swept-source optical coherence tomography (SS-OCT).

- DESIGN: Prospective cross-sectional study.

- Methods: Setting: Multicenter. Patient PopulaTION: Total of 143 diabetic eyes-27 with no diabetic retinopathy (DR), 47 with nonproliferative DR (NPDR), 51 with NPDR and diabetic macular edema (DME), and 18 with proliferative DR (PDR)—and 64 age-matched nondiabetic control eyes. OBSERVATION PROCEDURES: Complete ophthalmologic examination and $\overline{\text { SS-OCT }}$ imaging. En face SS-OCT images of the choroidal vasculature were binarized. MAIN OUTCOME MEAsurEs: CVD, calculated as the percent area occupied by choroidal vessels in the central macular region $(6-\mathrm{mm}$ diameter circle centered on the fovea), and throughout the posterior pole $(12 \times 9 \mathrm{~mm})$. The central macular CVV was calculated by multiplying the average CVD by macular area and choroidal thickness (obtained with SS-OCT automated software). Multilevel mixed linear models were performed for analyses.

- RESULTS: Compared to controls $(0.31 \pm 0.07)$, central macular CVD was significantly decreased by $9 \%$ in eyes with NPDR + DME (0.28 $\pm 0.06 ; \beta=-0.03$, $P=.02)$ and by $15 \%$ in PDR $(0.26 \pm 0.05$; $B=-0.04, P=.01)$. The central macular $C V V$ was significantly decreased by $19 \%$ in eyes with PDR $\left(0.020 \pm 0.005 \mathrm{~mm}^{3}, \beta=-0.01, P=.01\right)$ compared to controls $\left(0.025 \pm 0.01 \mathrm{~mm}^{3}\right)$.

- CONCLUSIONS: Choroidal vascular density and volume are significantly reduced in more advanced stages of diabetic retinopathy. New imaging modalities should allow further exploration of the contributions of choroidal vessel disease to diabetic eye disease pathogenesis, prognosis, and treatment response.



IABETIC RETINOPATHY (DR) IS A MAJOR CAUSE OF visual loss worldwide and is estimated to affect up to $35 \%$ of patients with diabetes. ${ }^{1}$ The pathogenesis of DR is primarily attributed to a dysregulation of the retinal vasculature, involving breakdown of the bloodretina barrier. ${ }^{2}$ Recent evidence, however, also points to the presence of a diabetic choroidopathy. ${ }^{3-7}$ It has also been hypothesized that choroidal vessel abnormalities might be responsible for decreased vision in DR, even in the absence of foveal changes in the retina. ${ }^{4}$

The development of the enhanced depth imaging (EDI) protocol for spectral-domain optical coherence tomography (SD-OCT) has enabled detailed noninvasive imaging of the choroid. Several studies have been published to assess choroidal thickness (CT) in DR, with somewhat conflicting results, but mostly demonstrating a decrease in CT. ${ }^{8-18}$ Recently, our group described a decreased CT in eyes with proliferative DR, using swept-source OCT (SS-OCT). ${ }^{19}$ SS-OCT seems to have several advantages for the assessment of the choroid. It uses a different light source (wavelength-tunable laser) and detection method such that longer wavelengths can be used. This allows for deeper penetration and lower scattering from the retinal pigment epithelium. ${ }^{20}$

In addition to CT, and to further assess the choroidal vasculature, the choroidal vascularity index $(\mathrm{CVI})$ has recently been introduced and proposed as a marker for vascular health of this layer. ${ }^{21-23}$ The CVI is obtained by binarizing EDI-OCT B-scan images, thus distinguishing lumens of choroidal vasculature from surrounding stroma. In healthy subjects, subfoveal CT was shown to be the 
only significant association with CVI. ${ }^{21}$ In DR, a reduced CVI has been recently noted. ${ }^{23}$

A similar index, the choroidal vascular density (CVD), can be obtained by binarizing high-resolution en face SS-OCT images. In control patients, this CVD has been shown to be significantly associated with advancing age and CT. ${ }^{24,25}$ CVD has been evaluated in other chorioretinal diseases, including age-related macular degeneration and central serous chorioretinopathy. ${ }^{26,27}$ Though there is evidence of localized choroidal microvascular changes in $\mathrm{DR},{ }^{28}$ to our knowledge CVD has not been previously assessed in this disease.

Here, we use SS-OCT en face images to analyze CVD and introduce choroidal vascular volume (CVV) as a global index (combining CVD and CT) in eyes with diabetes as compared to eyes from healthy nondiabetic subjects.

\section{METHODS}

- STUDY DESIGN: This study was conducted as part of a cross-sectional, observational, multicenter study on retinal imaging performed at the Retina Service, Massachusetts Eye and Ear (MEE), Harvard Medical School, Boston, Massachusetts, USA; and the Ophthalmology Department, Centro Hospitalar e Universitário de Coimbra (CHUC), Coimbra, Portugal, in collaboration with the Association for Innovation and Biomedical Research on Light and Image (AIBILI), Coimbra, Portugal. This research adhered to the tenets of the Declaration of Helsinki. The Institutional Review Boards of MEE and of AIBILI approved the study protocol. As required by law in Portugal, the Portuguese National Committee of Data Protection (CNPD) also approved this study. All included participants provided written informed consent.

- STUDY SUBJECTS: Consecutive diabetic patients were prospectively identified at CHUC/AIBILI and the MEE Retina Service, with the following exclusion criteria: refractive error greater than or equal to 6 diopters spherical equivalent; diagnosis of ocular hypertension or glaucoma with an optic nerve cup-to-disc ratio greater than 0.6 ; treatment in the 90 days prior to inclusion with laser capsulotomy, focal laser, panretinal photocoagulation, or intravitreal injections; any previous retinal surgery; diagnosis of other retinal or choroidal pathology, namely agerelated macular degeneration, vitreomacular traction, epiretinal membrane, macular hole, uveitis; systemic diseases that might affect CT, such as uncontrolled hypertension, systemic lupus erythematosus, anemia, leukemia, and obstructive sleep apnea; and decreased media transparency that precluded appropriate OCT imaging.

Simultaneously, nondiabetic subjects without any diagnosed vitreoretinal disease were also included as a control group. The same exclusion criteria were applied.
- ClINICAL STUDY PROTOCOL: Our study protocol has been described in detail by Laíns and associates. ${ }^{19}$ Briefly, all participants underwent complete ophthalmologic examination and their medical charts were reviewed to collect clinical variables, such as age, sex, and comorbidities, among others.

Diabetic eyes were categorized according to the Early Treatment Diabetic Retinopathy Study (ETDRS), ${ }^{29}$ as follows: no DR, no signs of DR; NPDR, nonproliferative DR without macular edema; NPDR + DME, NPDR with macular edema; and PDR, active proliferative DR or any previous treatment with panretinal photocoagulation (PRP).

- SWEPT-SOURCE OPTICAL COHERENCE TOMOGRAPHY IMAGING: At the same visit, all study subjects were imaged using the Topcon DRI OCT-1 Atlantis (Capelle aan den IJssel, The Netherlands). ${ }^{19}$ CT was obtained with the built-in software of the SS-OCT device (Topcon FastMap, version 9.12.003.04). User-independent thickness maps were created according to the conventional ETDRS grid.

For all subjects, an experienced, masked investigator (I.L., K.E.T., P.G., or J.G.) examined the position of the ETDRS grid, as well as the retinal and choroidal segmentations for all the obtained volume scans. Manual corrections were performed if the automated positioning or segmentation was deemed inaccurate. ${ }^{19}$

Finally, the obtained CT values in the 9 different ETDRS fields were registered. The average CT, defined as the arithmetic average of all sub-regions of the standard ETDRS grid, and the maximum CT, defined as the maximal choroidal thickness value of all sub-regions of the ETDRS grid, were recorded.

- CHOROIDAL VASCULAR DENSITY AND VOLUME: SS-OCT en face images of choroidal vasculature were obtained and flattened with the Bruch membrane (BM) as reference, using the en face tool included in the DRI OCT visualization software (Topcon, Tokyo, Japan). Images with substantial motion artifacts were excluded from analysis, as detailed in the Results section.

The image analysis procedure described herein is depicted in the Figure. The en face images were exported every $2.6 \mu \mathrm{m}$ from $\mathrm{BM}$ to the choroidal-scleral interface (CSI) and subsequently imported to ImageJ (National Institutes of Health, Bethesda, Maryland, USA) as an image stack. The image stack was converted to binary images using the auto-thresholding function of the software (ImageJ command "Image $>$ Adjust $>$ Threshold $>$ Apply") so as to distinguish the choroidal vasculature from choroidal stroma. The binarization of the en face image was done using the Otsu method, which is an automatic threshold selecting algorithm using gray-level histograms. ${ }^{30}$ Noise in the resulting binarized images was then removed (ImageJ command "Process $>$ Noise $>$ Remove Outliers"). The signal from the optic disc, retinal blood vessels, and any minor artifact was accounted for by creating a mask from the 


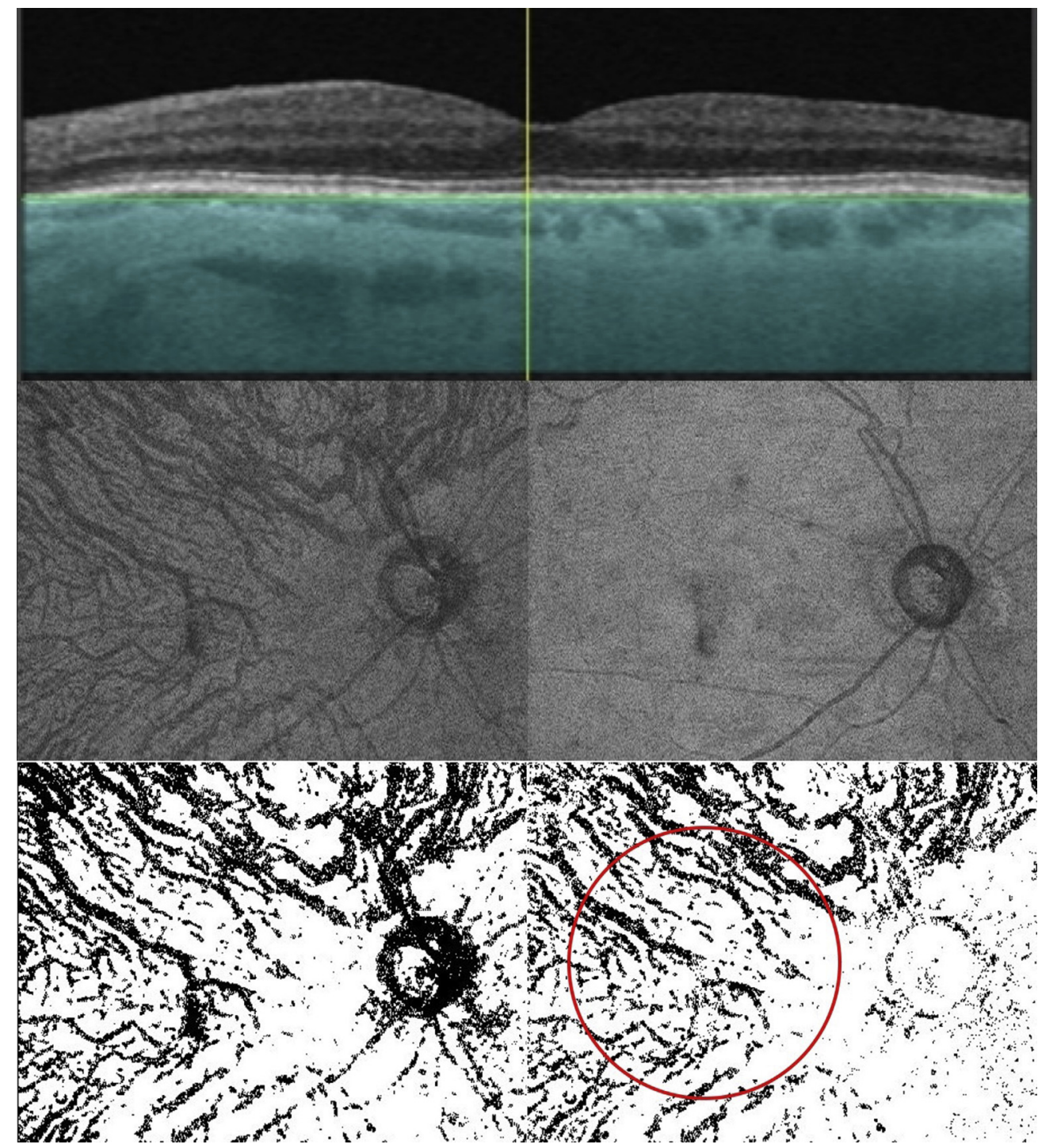

FIGURE. Representative example of swept source optical coherence tomography (SS-OCT) en face images and image processing. (Top) SS-OCT B-scan after flattening of the Bruch membrane and extraction of en face images from the Bruch membrane to the choroidal-scleral interface. (Middle left) Representative en face SS-OCT image $70 \mu \mathrm{m}$ below Bruch membrane; the optic disc and retinal vessels are visible in addition to the choroidal vasculature. (Bottom left) The same image after binarization. (Middle right) En face SS-OCT image at the level of the Bruch membrane showing optic nerve and retinal blood vessels that is used as a mask and applied to all subsequent images. (Bottom right) The same representative image after masking. The optic nerve and blood vessels are no longer visible after masking. The red circle is $6 \mathrm{~mm}$ in diameter centered on the fovea; this region is used for macular subanalysis.

image slice at the level of BM, and then applying that mask to all subsequent images, thereby eliminating any signal from those locations throughout the entire image stack (ImageJ command "Process > Image Calculator $>$ Multiply"). A 6-mm-diameter circular macular region centered on the fovea was cropped from the wider posterior pole image. Finally, the area occupied by choroidal vessels was analyzed (ImageJ command "Analyze > Measure"), and the choroidal vascular density was calculated as a percent area occupied by the choroidal vessels for each slice.

The average of the choroidal vascular densities of all image slices between the Bruch membrane and corresponding to the maximal CT was recorded as the average CVD. The overall average CVD was calculated from the whole $12 \times$ 9-mm wider posterior pole image, whereas the average macular CVD was calculated from the 6-mm-diameter circular macular region centered on the fovea. The macular CVV volume was calculated by multiplying the average CVD by the macular area and maximal CT.

- STATISTICAL ANALYSIS: The study population demographic, clinical, and structural characteristics were summarized with traditional descriptive methods. Considering the inclusion of both eyes of the same patients, 
TABLE 1. Demographic and Clinical Characteristics Relevant to Diabetes of Study Subjects Including Controls and Patients With Different Stages of Diabetic Retinopathy

\begin{tabular}{|c|c|c|c|c|c|}
\hline & Controls $(\mathrm{N}=64)$ & No $D R(N=27)$ & NPDR $(\mathrm{N}=47)$ & $\mathrm{NPDR}+\mathrm{DME}(\mathrm{N}=51)$ & $\operatorname{PDR}(\mathrm{N}=18)$ \\
\hline Age, years (mean $\pm S D$ ) & $63.9 \pm 13$ & $68.1 \pm 9.9$ & $65 \pm 9.8$ & $64.9 \pm 6.2$ & $67.2 \pm 10.1$ \\
\hline Female sex, $\mathrm{n}(\%)$ & $43(67 \%)$ & $5(19 \%)$ & $13(28 \%)$ & $19(37 \%)$ & 7 (39\%) \\
\hline Left eye, $\mathrm{n}(\%)$ & $34(53 \%)$ & $13(48 \%)$ & $20(43 \%)$ & $25(49 \%)$ & $11(61 \%)$ \\
\hline Spherical equivalent (mean \pm SD) & $-0.43 \pm 1.3$ & $0.66 \pm 1.2$ & $0.3 \pm 1.2$ & $-0.1 \pm 1.8$ & $+0.13 \pm 1.3$ \\
\hline Intraocular pressure (mean $\pm \mathrm{SD})$ & $16.8 \pm 1.6$ & $17.3 \pm 3.5$ & $16.7 \pm 2.5$ & $17.1 \pm 2.9$ & $16.2 \pm 3.5$ \\
\hline LogMAR BCVA (mean \pm SD) & $0.09 \pm 0.18$ & $0.04 \pm 0.15$ & $0.09 \pm 0.2$ & $0.3 \pm 0.27$ & $0.33 \pm 0.23$ \\
\hline \multicolumn{6}{|l|}{ Type of diabetes, n (\%) } \\
\hline Type 1 & - & $2(7 \%)$ & $4(9 \%)$ & $0(0 \%)$ & $0(0 \%)$ \\
\hline Type 2 & - & 25 (93\%) & $43(91 \%)$ & $51(100 \%)$ & $18(100 \%)$ \\
\hline \multicolumn{6}{|l|}{ Type of diabetes medication, $\mathrm{n}(\%)$} \\
\hline Oral & - & $24(89 \%)$ & 27 (57\%) & $14(27 \%)$ & $5(28 \%)$ \\
\hline Insulin & - & $3(11 \%)$ & $4(9 \%)$ & $2(4 \%)$ & $4(22 \%)$ \\
\hline Both & - & $0(0 \%)$ & $16(34 \%)$ & 35 (69\%) & $9(50 \%)$ \\
\hline Years with $\mathrm{DM}$ (mean $\pm \mathrm{SD}$ ) & - & $14.8 \pm 9.9$ & $20.2 \pm 7.2$ & $17 \pm 8.7$ & $23.3 \pm 13.6$ \\
\hline Hemoglobin A1C (mean \pm SD) & - & $6.4 \pm 0.9$ & $8.0 \pm 1.7$ & $8.1 \pm 2.1$ & $8.1 \pm 1.2$ \\
\hline DME at time of imaging, $\mathrm{n}(\%)$ & - & - & $0(0 \%)$ & $51(100 \%)$ & $9(50 \%)$ \\
\hline Eyes that received anti-VEGF injections, $\mathrm{n}(\%)$ & - & - & $2(4 \%)$ & $9(18 \%)$ & $7(39 \%)$ \\
\hline Total number injections (mean $\pm \mathrm{SD}$ ) & - & - & $5 \pm 2.8$ & $6.7 \pm 7.1$ & $2.6 \pm 1.6$ \\
\hline Months since last injection (mean $\pm \mathrm{SD}$ ) & - & - & $12 \pm 8.5$ & $9.3 \pm 3.7$ & $23 \pm 20$ \\
\hline Focal laser, $\mathrm{n}(\%)$ & - & - & $3(6 \%)$ & $20(39 \%)$ & $8(44 \%)$ \\
\hline Number of sessions (mean \pm SD) & - & - & $1.3 \pm 0.6$ & $2.1 \pm 2.0$ & $2.3 \pm 0.5$ \\
\hline Total spots (mean \pm SD) & - & - & $118 \pm 6.4$ & $130 \pm 110$ & $134 \pm 62$ \\
\hline Months since last (mean $\pm \mathrm{SD}$ ) & - & - & $6 \pm 5$ & $35 \pm 32$ & $23 \pm 19$ \\
\hline PRP, n (\%) & - & - & - & - & $13(72 \%)$ \\
\hline Number of sessions (mean \pm SD) & - & - & - & - & $4.9 \pm 3.9$ \\
\hline Total spots (mean \pm SD) & - & - & - & - & $5300 \pm 4000$ \\
\hline Months since last (mean $\pm \mathrm{SD}$ ) & - & - & - & - & $24 \pm 25$ \\
\hline
\end{tabular}

for all analyses we used multilevel mixed-effect linear models. By definition, these models are appropriate for research designs where data for participants is nested. ${ }^{31}$ In this study, the units of analysis were considered to be the eyes (at a lower level), which are nested within patients-contextual/aggregate units (at a higher level).

Using these models, our statistical approach was centered on overall CVD (ie, the entire wider posterior pole image), macular CVD (ie, only the 6-mm-diameter circle centered on the fovea), and macular CVV (ie, only the 6-mm-diameter circle centered on the fovea). We started with univariate analyses for all potential covariates (Table 1 ) and all the parameters with a $P$ value $\leq .250$ were included in the initial multivariate model. Other parameters that did not meet this statistical criterion but with known clinical relevance for choroidal vasculature (such as spherical equivalent) were also considered. ${ }^{32-35} \mathrm{~A}$ backward (step-down) elimination procedure was then used to achieve the multivariate models presented.
All statistics were performed using Stata version 12.1 (StataCorp LP, College Station, Texas, USA), and P values $<.05$ were considered statistically significant.

\section{RESULTS}

- DEMOGRAPHICS AND CLINICAL CHARACTERISTICS: A total of 233 eyes of 128 subjects were included in the study (157 eyes of 90 diabetic subjects and 76 eyes of 38 control subjects). Thirty percent of the eyes were included at MEE ( $\mathrm{n}=71$ eyes) and the remaining at CHUC/AIBILI $(\mathrm{n}=162$ eyes). Owing to significant motion artifacts that precluded appropriate image analysis, we then excluded 12 control eyes and 14 diabetic eyes. The overall clinical and demographic characteristics for both groups are summarized in Table 1. 
ABLE 2. Summary of Choroidal Vascular Indices and Choroidal Thickness Values in Control Eyes and in Eyes With Different Stages of Diabetic Retinopathy

\begin{tabular}{lccccc}
\hline & Controls $(\mathrm{N}=64)$ & No $\mathrm{DR}(\mathrm{N}=27)$ & NPDR $(\mathrm{N}=47)$ & NPDR $+\mathrm{DME}(\mathrm{N}=51)$ & PDR $(\mathrm{N}=18)$ \\
\hline Average CVD (mean $\pm \mathrm{SD})$ & $0.23 \pm 0.03$ & $0.24 \pm 0.03$ & $0.22 \pm 0.03$ & $0.22 \pm 0.03$ & $0.20 \pm 0.02$ \\
Average macular CVD (mean $\pm \mathrm{SD})$ & $0.31 \pm 0.07$ & $0.34 \pm 0.05$ & $0.28 \pm 0.06$ & $0.28 \pm 0.06$ & $0.26 \pm 0.05$ \\
Macular CVV (mean $\left.\pm \mathrm{SD}, \mathrm{mm}^{3}\right)$ & $0.025 \pm 0.01$ & $0.028 \pm 0.01$ & $0.021 \pm 0.01$ & $0.023 \pm 0.01$ & $0.020 \pm 0.01$ \\
Average macular CT (mean $\pm \mathrm{SD}, \mathrm{mm})$ & $201 \pm 82$ & $219 \pm 49$ & $181 \pm 75$ & $189 \pm 63$ & $153 \pm 45$ \\
\hline
\end{tabular}

$\mathrm{CT}=$ choroidal thickness; CVD = choroidal vascular density; CVV = choroidal vascular volume; $\mathrm{DME}=$ diabetic macular edema; $\mathrm{DR}=$ diabetic retinopathy; NPDR = nonproliferative diabetic retinopathy; PDR = proliferative diabetic retinopathy.

- CHOROIDAL VASCULAR INDICES AND THICKNESS: Choroidal vascular indices-overall average CVD, average macular CVD, macular CVV, and choroidal thickness stratified by stage of DR-are displayed in Table 2 . Compared to control eyes, the overall average CVD was significantly reduced by $6 \%$ in eyes with NPDR, by $7 \%$ in eyes with NPDR + DME, and by $12 \%$ in eyes with PDR. Similarly, the average macular CVD was significantly reduced by $9 \%$ in eyes with NPDR + DME and by $15 \%$ in eyes with PDR compared to control eyes. Finally, the macular CVV was significantly reduced by $19 \%$ in eyes with PDR compared to control eyes.

- UNIVARIATE ANALYSES: Univariate analysis revealed that increasing severity of diabetic retinopathy was significantly associated with decreased choroidal vascular indices. Specifically, when compared to the control group, eyes with NPDR presented decreased overall average CVD $(\beta=-0.01, P=.034)$; eyes with NPDR + DME also showed decreased overall average CVD $(\beta=-0.02$, $P=.007)$ and average macular CVD $(\beta=-0.02$, $P=.033)$; and eyes with PDR presented a decreased overall average $\operatorname{CVD}(\beta=-0.03, P=.005)$, average macular $\operatorname{CVD}(\beta=-0.04, P=.035)$, and macular $\mathrm{CVV}$ $(\beta=-0.007, P=.022)$ (Table 3$)$.

We also assessed the influence of potential confounders in these outcomes. Our results revealed that sex, spherical equivalent, and intraocular pressure were not significant predictors of average CVD, average macular CVD, and macular CVV (Table 3). Increasing age was significantly associated with a reduction in our choroidal vascular indices.

A prior history of anti-vascular endothelial growth factor (anti-VEGF) injections was not a statistically significant predictor of overall average CVD, average macular CVD, or macular CVV $(P>.7)$ in patients with diabetes. In the subgroup of patients with PDR, we also compared choroidal vessel indices among patients that had undergone PRP to those who had not. Our results revealed that there was no statistically significant difference in overall average CVD $(\beta=-0.02, P=.123)$, average macular CVD $(\beta=0.03, P=.227)$, and macular CVV $(\beta=0.002, P=.323)$.
- MUlTIVARIATE ANALYSES: Results of multivariate analyses of overall average CVD, average macular CVD, and macular CVV comparing diabetic eyes to control eyes are summarized in Table 4. As detailed, we included age in the multivariate models owing to its significance on univariate analysis. Despite not presenting a significant association in our univariate assessments, we also included spherical equivalent owing to the well-established influence of this parameter in choroidal vasculature. ${ }^{32}$

Multivariate analysis revealed that increasing severity of diabetic retinopathy was significantly associated with decreased choroidal vascular indices. Specifically, the presence of NPDR was significantly associated with decreased overall average CVD $(\beta=-0.01, P=.041)$. Eyes with NPDR + DME presented decreased overall average CVD $(\beta=-0.02, P=.009)$ and average macular CVD $(\beta=-0.03, P=.023)$. Finally, eyes with PDR showed decreased overall average $\operatorname{CVD}(\beta=-0.02, P=.005)$, average macular $\operatorname{CVD}(\beta=-0.04, P=.011)$, and macular $\operatorname{CVV}(\beta=-0.007, P=.011)$.

\section{DISCUSSION}

WE PRESENT A MULTICENTER CROSS-SECTIONAL STUDY USing SS-OCT en face images to analyze choroidal vascularity in different stages of DR. Building upon previous CT work, we introduce CVD and CVV as new quantitative parameters of the choroidal vasculature. Our results revealed that, even after accounting for confounding factors, diabetic eyes with DME or PDR demonstrated a reduced CVD, as compared to controls. Additionally, eyes with PDR also demonstrated reduced CVV. These findings are in line with the notion that vascular abnormalities accumulate with the severity of DR, which is reflected in the choroidal vascular indices that we assessed.

Our study adds to the growing body of literature on diabetic choroidopathy ${ }^{36}$ and, to the best of our knowledge, is the first to examine CVD in different stages of DR. Much of the previous literature has focused on CT, with most studies showing some degree of choroidal thinning in 
TABLE 3. Univariate Multilevel Mixed Linear Regression Analysis Comparing Choroidal Vascular Indices in Control Eyes to Eyes With Different Stages of Diabetic Retinopathy

\begin{tabular}{|c|c|c|c|c|c|c|c|c|c|}
\hline & \multicolumn{3}{|c|}{ Average CVD } & \multicolumn{3}{|c|}{ Average Macular CVD } & \multicolumn{3}{|c|}{ Macular CVV } \\
\hline & $\beta$ Coefficient & $95 \% \mathrm{Cl}$ & $P$ Value & $\beta$ Coefficient & $95 \% \mathrm{Cl}$ & $P$ Value & $\beta$ Coefficient & $95 \% \mathrm{Cl}$ & $P$ Value \\
\hline Age & -0.001 & -0.002 to -0.001 & $<.0005^{\star}$ & -0.003 & -0.004 to -0.002 & $<.0005^{\star}$ & -0.0005 & -0.0006 to -0.0003 & $<.0005^{\star}$ \\
\hline Sex & -0.0007 & -0.01 to 0.01 & .936 & -0.007 & -0.03 to 0.02 & .543 & -0.001 & -0.005 to 0.003 & .549 \\
\hline SE & -0.0004 & -0.003 to 0.002 & .762 & 0.003 & -0.003 to 0.009 & .308 & 0.0005 & -0.0004 to 0.001 & .286 \\
\hline IOP & -0.00003 & -0.001 to 0.001 & .962 & 0.002 & -0.001 to 0.004 & .181 & 0.0001 & -0.0002 to 0.0005 & .461 \\
\hline Left eye & -0.003 & -0.007 to 0.001 & .205 & -0.002 & -0.001 to 0.006 & .650 & -0.00003 & -0.001 to 0.001 & .951 \\
\hline Years with DM & -0.0001 & -0.0008 to 0.0006 & .756 & -0.0007 & -0.002 to 0.0007 & .351 & -0.00007 & -.00003 to 0.00001 & .524 \\
\hline $\mathrm{HbA1c}$ & 0.0008 & -0.003 to 0.004 & .656 & 0.002 & -0.005 to 0.009 & .537 & 0.0004 & -0.0006 to 0.001 & .410 \\
\hline DM med type & -0.0009 & -0.007 to 0.006 & .780 & -0.009 & -.02 to 0.004 & .170 & 0.0004 & -0.002 to 0.002 & .721 \\
\hline \multicolumn{10}{|c|}{ Level of DR (as compared to controls) } \\
\hline No DR & -0.002 & -0.01 to 0.02 & .817 & 0.01 & -0.02 to 0.05 & .375 & -0.0005 & -0.005 to 0.005 & .859 \\
\hline NPDR & -0.01 & -0.03 to -0.001 & $.034^{*}$ & -0.02 & -0.05 to 0.002 & .073 & -0.003 & -0.007 to 0.001 & .157 \\
\hline NPDR + DME & -0.02 & -0.03 to 0.005 & $.007^{\star}$ & -0.03 & -0.05 to -0.002 & $.033^{\star}$ & -0.003 & -0.008 to 0.0007 & .108 \\
\hline PDR & -0.03 & -0.05 to 0.008 & $.005^{\star}$ & -0.04 & -0.08 to -0.003 & $.035^{\star}$ & -0.007 & -0.01 to -0.001 & $.022^{*}$ \\
\hline $\begin{array}{r}\mathrm{Cl}=\text { confider } \\
\text { ular edema; } \mathrm{DR} \\
\text { both); NPDR }= \\
\text { Significant } P\end{array}$ & $\begin{array}{l}=\text { diabetic } \\
\text { Ionprolifer }\end{array}$ & tinopathy; $\mathrm{HbA} 1 \mathrm{c}$ & emoglob & $=$ promer & alabetic retin & ny, s & $\begin{array}{l}=\text { medic } \\
=\text { spheric }\end{array}$ & $\begin{array}{l}\text { on (insulin, oral ant } \\
\text { equivalent. }\end{array}$ & $\begin{array}{l}\text { tic mac- } \\
\text { etics, or }\end{array}$ \\
\hline
\end{tabular}

TABLE 4. Multilevel Mixed Multivariate Linear Regression Analysis Comparing Choroidal Vascular Indices in Control Eyes to Eyes With Different Stages of Diabetic Retinopathy

\begin{tabular}{|c|c|c|c|c|c|c|c|c|c|}
\hline & \multicolumn{3}{|c|}{ Average CVD } & \multicolumn{3}{|c|}{ Average Macular CVD } & \multicolumn{3}{|c|}{ Macular CVV } \\
\hline & $\beta$ Coefficient & $95 \% \mathrm{Cl}$ & $P$ Value & $\beta$ Coefficient & $95 \% \mathrm{Cl}$ & $P$ Value & $\beta$ Coefficient & $95 \% \mathrm{Cl}$ & $P$ Value \\
\hline Age & -0.002 & -0.002 to -0.001 & $<.0005^{\star}$ & -0.002 & -0.004 to -0.002 & $<.0005^{\star}$ & -0.0005 & -0.0007 to -0.0004 & $<.0005^{\star}$ \\
\hline SE & 0.001 & -0.002 to 0.003 & .569 & 0.005 & 0 to 0.011 & $.048^{\star}$ & 0.001 & 0.0002 to 0.002 & $.011^{*}$ \\
\hline \multicolumn{10}{|c|}{ Level of DR (as compared to controls) } \\
\hline No DR & 0.007 & -0.007 to 0.02 & .344 & 0.03 & -0.004 to 0.06 & .087 & 0.001 & -0.003 to 0.005 & .654 \\
\hline NPDR & -0.01 & -0.02 to -0.0005 & $.041^{*}$ & -0.02 & -0.05 to 0.002 & .078 & -0.003 & -0.006 to 0.009 & .143 \\
\hline NPDR + DME & -0.02 & -0.03 to -0.004 & $.009^{\star}$ & -0.03 & -0.05 to -0.004 & $.023^{\star}$ & -0.002 & -0.006 to 0.001 & .175 \\
\hline PDR & -0.02 & -0.04 to -0.007 & $.005^{\star}$ & -0.04 & -0.08 to -0.01 & $.011^{*}$ & -0.007 & -0.01 to -0.002 & $.011^{\star}$ \\
\hline
\end{tabular}

$\mathrm{Cl}=$ confidence interval; $\mathrm{CVD}=$ choroidal vascular density; $\mathrm{CVV}=$ choroidal vascular volume; $\mathrm{DME}=$ diabetic macular edema; $\mathrm{DR}=$ diabetic retinopathy; NPDR = nonproliferative diabetic retinopathy; PDR = proliferative diabetic retinopathy; SE = spherical equivalent.

Significant $P$ values $(<.05)$ are indicated by asterisk $\left(^{*}\right)$.

eyes with diabetes, especially with more severe diabetic retinopathy..$^{8-17,19}$ The exact pathophysiology of diabetic choroidopathy, as well as its relation with diabetic retinopathy, remains to be established. Diabetic choroidopathy may occur as a primary manifestation of systemic diabetic disease independent of diabetic retinopathy. Alternatively, it can be secondary to diabetic changes in the retina or treatment interventions, either ocular or systemic. Notably, we observed that in diabetic patients without diabetic retinopathy, there were no significant differences in choroidal vascular indices when compared to controls. This suggests the possibility that diabetic choroidopathy may occur simultaneously with or as a result of diabetic retinopathy, rather than preceding it, though further study of this particular subset of patients is needed. Elucidating the relationship between diabetic choroidopathy and retinopathy is essential for a more complete understanding of diabetic eye disease.

Qualitatively, changes in the choroidal vascular architecture have been observed in histologic studies, including basement membrane thickening of small choroidal blood 
vessels, luminal narrowing of capillaries, microaneurysms, dropout of the choriocapillaris, and choroidal neovascularization. ${ }^{3,36}$ Angiographic studies using fluorescein angiography and indocyanine green angiography have also shown irregular filling of the choriocapillaris, early hypofluorescent spots representing filling delays, and late hyperfluorescent spots representing intrachoroidal microvascular abnormalities. ${ }^{37,38}$

Other studies have devised different strategies to quantitatively characterize the choroidal vasculature from OCT images in DR. For instance, one group investigated the largest hyporeflective lumen on EDI SD-OCT B-scans as a proxy for choroidal vascularity. ${ }^{39}$ The authors described that the mean diameter of the largest hyporeflective lumen was reduced in patients with diabetes. However, though this was a large study, there is no mention of stratifying patients into different stages of DR. Similarly, a second group also found a reduced CVI in DR using EDI SD-OCT images, but DR stage was not specified. ${ }^{23}$

In our study, in contrast to the prior literature, we used SS-OCT to define CVD. SS-OCT offers several advantages, particularly higher-resolution imaging of the choroid, deeper signal penetration by its longer wavelength, and automatic choroidal thickness measurements. Thus, we assert that the CVD is preferable to the CVI and largest hyporeflective lumen as an index for choroidal vascularity. We are also not aware of recent studies measuring CVD from en face SS-OCT images in the context of DR. Previous authors have used SS-OCT to qualitatively describe, in eyes with diabetes, the presence of focal narrowing of choroidal blood vessels in the Haller layer, as well as the presence of vessels ending prematurely in the superficial or middle portion of the Haller layer (referred to as "vascular stumps"). ${ }^{28}$ These authors did note a statistically significant trend toward increased proportions of "vascular stumps" in eyes with more advanced stages of diabetic retinopathy.

As the CVV is derived from both the CVD and CT, it is likely that statistically significant reductions in CT contributed to significant reductions in CVV in PDR. For NPDR and NPDR + DME, the differences in CT were not statistically significant, which may be owing to a true lack of differences, or to our study design, considering the heterogeneity of these groups (subjects were not further subclassified into mild, moderate, and severe NPDR). In NPDR without DME, overall CVD was significantly reduced but macular CVD was not, suggesting that notable submacular choroidal pathology only becomes present in later stages of DR, whereas diffuse choroidal changes may already be present in early stages of DR.

Our study has several strengths. It was a multicenter study, where we prospectively recruited a relatively large number of patients; patients with DR were subdivided by stage of DR; our data were derived from high-resolution SS-OCT imaging as opposed to SD-OCT; and we performed multivariate analysis, accounting for confounding factors, such as age and spherical equivalent. Additionally, we devised a masking procedure in the image-processing algorithm to eliminate the optic disc and retinal vessels from the binarized images. This allowed for quantification of a larger area of the choroid than what had previously been reported. Our results were consistent among different vascular indices, which serves to strengthen the internal validity of our study. Furthermore, based on our experience, en face SS-OCT may actually be superior to OCT angiography for choroidal vessel analysis, given inherent artifacts and limitations of the current OCT angiography technology. ${ }^{40}$

Our study does have some limitations. Though there was a small number of patients with type 1 diabetes in our study, univariate and multivariate regression analyses did not reveal a statistically significant association between diabetes type and CVD or CVV indices. Despite baseline differences in some clinical characteristics, such as sex, medication type, and years with diabetes, these variables were analyzed in our regression models and were not found to have statistically significant associations with our outcomes (CVD and CVV). We did include patients who had previously undergone PRP and intravitreal antiVEGF injections, which is known to affect CT. ${ }^{41-44}$ To account for this, we excluded eyes that had PRP or antiVEGF injections 3 months prior to study inclusion, to minimize any acute changes associated with treatment, but this might have affected the results. Reassuringly, we found no statistically significant difference in the choroidal vascular indices of eyes with PDR that had undergone PRP in comparison to those who had not had received this treatment. We also found that history of anti-VEGF injection was not statistically associated with our CVD or CVV indices. It is possible that the inclusion of patients with a history of hypertension could have affected our results for CVD and CVV, which may represent a limitation of this study. In evaluation of the associations between choroidal vascular indices and stages of diabetic retinopathy, multiple tests were completed that could have led to falsepositive results. Finally, our study was not adequately powered to detect statistically significant differences in the subgroup of patients with PDR with and without DME. Additional large prospective studies examining CVD and CVV through diabetic retinopathy should help us better understand the choroid's role in diabetic eye disease.

In conclusion, we used swept-source OCT en face imaging to identify reductions in choroidal vessel density and volume in diabetic eyes; increasing reductions were observed with increasing diabetic retinopathy severity. Additional work with CVV and CVD in diabetic eye disease may contribute to improved understanding of pathogenesis, prognosis, and treatment of diabetic retinopathy. 


\section{REFERENCES}

1. Yau JWY, Rogers SL, Kawasaki R, et al. Global prevalence and major risk factors of diabetic retinopathy. Diabetes Care 2012;35(3):556-564.

2. Antonetti DA, Klein R, Gardner TW. Diabetic retinopathy. N Engl J Med 2012;366(13):1227-1239.

3. Hidayat AA, Fine BS. Diabetic choroidopathy. Light and electron microscopic observations of seven cases. Ophthalmology 1985;92(4):512-522.

4. Cao J, McLeod S, Merges CA, Lutty GA. Choriocapillaris degeneration and related pathologic changes in human diabetic eyes. Arch Ophthalmol 1998;116(5):589-597.

5. Fryczkowski AW, Sato SE, Hodes BL. Changes in the diabetic choroidal vasculature: scanning electron microscopy findings. Ann Ophthalmol 1988;20(8):299-305.

6. Nagaoka T, Kitaya N, Sugawara R, et al. Alteration of choroidal circulation in the foveal region in patients with type 2 diabetes. Br J Ophthalmol 2004;88(8):1060-1063.

7. Schocket LS, Brucker AJ, Niknam RM, Grunwald JE, DuPont J, Brucker AJ. Foveolar choroidal hemodynamics in proliferative diabetic retinopathy. Int Ophthalmol 2004; 25(2):89-94.

8. Wang S, Lin S, Zheng Y, et al. [Association of choroidal thickness with diabetic retinopathy at different stages]. Zhonghua Yi Xue Za Zhi 2015;95(32):2584-2588.

9. Lee HK, Lim JW, Shin MC. Comparison of choroidal thickness in patients with diabetes by spectral-domain optical coherence tomography. Korean J Ophthalmol 2013;27(6):433.

10. Vujosevic S, Martini F, Cavarzeran F, Pilotto E, Midena E. Macular and peripapillary choroidal thickness in diabetic patients. Retina 2012;32(9):1781-1790.

11. Querques G, Lattanzio R, Querques L, et al. Enhanced depth imaging optical coherence tomography in type 2 diabetes. Invest Ophthalmol Vis Sci 2012;53(10):6017.

12. Esmaeelpour M, Považay B, Hermann B, et al. Mapping choroidal and retinal thickness variation in type 2 diabetes using three-dimensional 1060-nm optical coherence tomography. Invest Ophthalmol Vis Sci 2011;52(8):5311.

13. Unsal E, Eltutar K, Zirtiloglu S, Dincer N, Ozdogan Erkul S, Gungel H. Choroidal thickness in patients with diabetic retinopathy. Clin Ophthalmol 2014;8:637.

14. Regatieri CV, Branchini L, Carmody J, Fujimoto JG, Duker JS. Choroidal thickness in patients with diabetic retinopathy analyzed by spectral-domain optical coherence tomography. Retina 2012;32(3):563-568.

15. Gerendas BS, Waldstein SM, Simader C, et al. Three-dimensional automated choroidal volume assessment on standard spectral-domain optical coherence tomography and correlation with the level of diabetic macular edema. Am J Ophthalmol 2014;158(5):1039-1048.e1.

16. Galgauskas S, Laurinaviciute G, Norvydaite D, Stech S, Ašoklis R. Changes in choroidal thickness and corneal parameters in diabetic eyes. Eur J Ophthalmol 2016;26(2): 163-167.

17. Sudhalkar A, Chhablani J, Venkata A, Raman R, Rao PS, Jonnadula G. Choroidal thickness in diabetic patients of Indian ethnicity. Indian J Ophthalmol 2015;63(12):912.
18. Adhi M, Brewer E, Waheed NK, Duker JS. Analysis of morphological features and vascular layers of choroid in diabetic retinopathy using spectral-domain optical coherence tomography. JAMA Ophthalmol 2013;131(10):1267-1274.

19. Laíns I, Talcott KE, Santos AR, et al. Choroidal thickness in diabetic retinopathy assessed with swept-source optical coherence tomography. Retina 2017, https://doi.org/10. 1097/IAE.00000000000001516, [Epub ahead of print].

20. Ferrara D, Mohler KJ, Waheed N, et al. En face enhanceddepth swept-source optical coherence tomography features of chronic central serous chorioretinopathy. Ophthalmology 2014;121(3):719-726.

21. Agrawal R, Gupta P, Tan K-A, Cheung CMG, Wong T-Y, Cheng C-Y. Choroidal vascularity index as a measure of vascular status of the choroid: measurements in healthy eyes from a population-based study. Sci Rep 2016;6:21090.

22. Agrawal R, Salman M, Tan K-A, et al. Choroidal vascularity index (CVI) - a novel optical coherence tomography parameter for monitoring patients with panuveitis? PLoS One 2016; 11(1):e0146344.

23. Tan K-A, Laude A, Yip V, Loo E, Wong EP, Agrawal R. Choroidal vascularity index - a novel optical coherence tomography parameter for disease monitoring in diabetes mellitus? Acta Ophthalmol 2016;94(7):e612-e616.

24. Wang Q, Chan S, Yang JY, et al. Vascular density in retina and choriocapillaris as measured by optical coherence tomography angiography. Am J Ophthalmol 2016;168:95-109.

25. Fujiwara A, Morizane Y, Hosokawa M, et al. Factors affecting choroidal vascular density in normal eyes: quantification using en face swept-source optical coherence tomography. Am J Ophthalmol 2016;170:1-9.

26. Kuroda Y, Oto S, Yamashiro K, et al. Increased choroidal vascularity in central serous chorioretinopathy quantified using swept-source optical coherence tomography. Am J Ophthalmol 2016;169:199-207.

27. Zheng F, Gregori G, Schaal KB, et al. Choroidal thickness and choroidal vessel density in nonexudative age-related macular degeneration using swept-source optical coherence tomography imaging. Invest Ophthalmol Vis Sci 2016;57(14): 6256.

28. Murakami T, Uji A, Suzuma K, et al. In vivo choroidal vascular lesions in diabetes on swept-source optical coherence tomography. PLoS One 2016;11(8):e0160317.

29. Early Treatment Diabetic Retinopathy Study Research Group. Early photocoagulation for diabetic retinopathy. ETDRS report number 9. Ophthalmology 1991;98(5 Suppl): 766-785.

30. Otsu N. A threshold selection method from gray-level histograms. IEEE Trans Syst Man Cybern 1979;9(1):62-66.

31. Burton P, Gurrin L, Sly P. Extending the simple linear regression model to account for correlated responses: an introduction to generalized estimating equations and multi-level mixed modelling. Stat Med 1998;17(11):1261-1291.

32. Sanchez-Cano A, Orduna E, Segura F, et al. Choroidal thickness and volume in healthy young white adults and the relationships between them and axial length, ammetropy and sex. Am J Ophthalmol 2014;158(3):574-583.e1. 
33. Fujiwara T, Imamura Y, Margolis R, Slakter JS, Spaide RF. Enhanced depth imaging optical coherence tomography of the choroid in highly myopic eyes. Am J Ophthalmol 2009; 148(3):445-450.

34. Wakatsuki Y, Shinojima A, Kawamura A, Yuzawa M. Correlation of aging and segmental choroidal thickness measurement using swept source optical coherence tomography in healthy eyes. PLoS One 2015;10(12):e0144156.

35. Wang J, Gao X, Huang W, et al. Swept-source optical coherence tomography imaging of macular retinal and choroidal structures in healthy eyes. BMC Ophthalmol 2015;15(1):122.

36. Melancia D, Vicente A, Cunha JP, Abegão Pinto L, Ferreira J. Diabetic choroidopathy: a review of the current literature. Graefes Arch Clin Exp Ophthalmol 2016;254(8): 1453-1461.

37. Weinberger D, Kramer M, Priel E, Gaton DD, Axer-Siegel R, Yassur Y. Indocyanine green angiographic findings in nonproliferative diabetic retinopathy. Am J Ophthalmol 1998;126(2): 238-247.

38. Hua R, Liu L, Wang X, Chen L. Imaging evidence of diabetic choroidopathy in vivo: angiographic pathoanatomy and choroidal-enhanced depth imaging. PLoS One 2013;8(12): e83494.

39. Verma A, Nagpal M, Mehrotra N. In vivo assessment of choroid in diabetic retinopathy by enhanced depth imaging in spectral domain optical coherence tomography. Asia Pac J Ophthalmol (Phila) 2016;5(5):319-323.

40. Spaide RF, Fujimoto JG, Waheed NK. Image artifacts in optical coherence tomography angiography. Retina 2015; 35(11):2163-2180.

41. Yiu G, Manjunath V, Chiu SJ, Farsiu S, Mahmoud TH. Effect of anti-vascular endothelial growth factor therapy on choroidal thickness in diabetic macular edema. Am J Ophthalmol 2014;158(4):745-751.e2.

42. Zhu Y, Zhang T, Wang K, Xu G, Huang X. Changes in choroidal thickness after panretinal photocoagulation in patients with type 2 diabetes. Retina 2015;35(4):695-703.

43. Okamoto M, Matsuura T, Ogata N. Effects of panretinal photocoagulation on choroidal thickness and choroidal blood flow in patients with severe nonproliferative diabetic retinopathy. Retina 2016;36(4):805-811.

44. Zhang Z, Meng X, Wu Z, et al. Changes in choroidal thickness after panretinal photocoagulation for diabetic retinopathy: a 12-week longitudinal study. Invest Ophthalmol Vis Sci 2015;56(4):2631. 